\title{
Disruption of kif3a results in defective osteoblastic differentiation in dental mesenchymal stem/precursor cells via the Wnt signaling pathway
}

\author{
SICONG JIANG ${ }^{1-3}$, GUOQING CHEN ${ }^{2,3}$, LIAN FENG $^{2,3}$, ZONGTING JIANG $^{2,3}$, \\ MEI YU ${ }^{2,3}$, JINKU BAO $^{1}$ and WEIDONG TIAN ${ }^{2,3}$ \\ ${ }^{1}$ School of Life Sciences and Key Laboratory of Bio-Resources and Eco-Environment, \\ Ministry of Education, Sichuan University, Chengdu, Sichuan $610064 ;{ }^{2}$ State Key Laboratory of Oral Diseases; \\ ${ }^{3}$ National Engineering Laboratory for Oral Regenerative Medicine, West China Hospital of Stomatology, \\ Sichuan University, Chengdu, Sichuan 610041, P.R. China
}

Received April 9, 2015; Accepted February 22, 2016

DOI: $10.3892 / \mathrm{mmr} .2016 .5508$

\begin{abstract}
The anterograde intraflagellar transport motor protein, kif3a, regulates the integrity of primary cilia and various cellular functions, however, the role of kif3a in dental mesenchymal stem/precursor cell differentiation remains to be fully elucidated. In the present study, the expression of kif3a was knocked down in human dental follicle cells (hDFCs) and human dental pulp cells (hDPCs) using short hairpin RNA. The results of subsequent immunofluorescence revealed that knocking down kif3a resulted in the loss of primary cilia, which led to impairment of substantial mineralization and expression of the differentiation-associated markers, including alkaline phosphatase, Runt-related transcription factor 2, dentin matrix protein 1 and dentin sialophosphoprotein in the hDFCs and hDPCs. The results of reverse transcription-quantitative polymerase chain reaction and western blot analyses showed that the expression levels of Wnt3a-mediated active $\beta$-catenin and lymphoid enhancer-binding factor 1 were attenuated, whereas the expression of phosphorylated glycogen synthase kinase $3 \beta$ was enhanced, in the kif3a-knockdown cells. In addition, exogenous Wnt3a partially rescued osteoblastic differentiation in the hDFCs and hDPCs. These results demonstrated that inhibition of kif3a in the hDFCs and hDPCs disrupted
\end{abstract}

Correspondence to: Professor Jinku Bao, School of Life Sciences and Key Laboratory of Bio-Resources and Eco-Environment, Ministry of Education, Sichuan University, 29 Wangjiang Road, Chengdu, Sichuan 610064, P.R. China

E-mail: baojinku@scu.edu.cn

Professor Weidong Tian, State Key Laboratory of Oral Diseases, West China Hospital of Stomatology, Sichuan University, 14, Third Section, Renmin South Road, Chengdu, Sichuan 610041, P.R. China E-mail: drtwd@sina.com

Key words: primary cilia, kif3a, dental follicle cells, dental pulp cells, osteoblastic differentiation, Wnt signaling primary cilia formation and/or function, and indicated that kif3a is important in the differentiation of hDFCs and hDPCs through the Wnt pathway. These findings not only enhance current understanding of tooth development and diseases of tooth mineralization, but also indicate possible strategies to regulate mineralization during tooth repair and regeneration.

\section{Introduction}

Stem cell treatment offers a promising approach in providing an advanced and reliable therapeutic strategy for tissue regeneration and disease therapy. Our previous studies, and those of others, have demonstrated that mesenchymal stem cells derived from dental tissues, including dental follicle stem cells (DFCs) and dental pulp stem cells (DPCs), can be used to successfully regenerate or repair bone, dentin and tooth roots (1-3). DFCs are derived from the neural crest and give rise to the periodontal ligament. DPCs are derived from the dental pulp and are also considered to originate from the cranial neural crest. These cells are multipotent and can differentiate into various cell types, including osteoblasts, adipocytes and neurons (4-6). Of note, these two types of cells can be readily isolated from extracted third molars, which are usually otherwise discarded as medical waste. Therefore, stem cell-derived dental tissues, including DFCs and DPCs, represent an alternative source of stem cells for tissue regeneration. However, the underlying mechanisms controlling the fate of these stem cells remain to be fully elucidated.

The kif3a protein is a member of the kinesin-2 family of motor proteins, which is associated with the intraflagellar transport (IFT) system of primary cilia, is an essential system for the maintenance of ciliogenesis and ciliary function $(7,8)$, and is key in the development of several organs, including the bone, brain and the thyroid gland (9-11). Previously, studies have provided evidence that kif3a is involved in the maturation of osteoblasts and osteoblastic differentiation of mesenchymal stem cells $(11,12)$. In particular, selective deletion of kif3a in osteoblasts of kif3a ${ }^{\mathrm{Oc}-\mathrm{cKO}}$ mice impairs postnatal bone formation through multiple pathways, including Wnt signaling, similar to 
the phenotype exhibited following cilia deletion by other IFT proteins (13), indicating that kif3a is involved in regulating Wnt signaling in a cilia-associated manner (11). Furthermore, another IFT protein, ofd1, is expressed in the dental mesenchyma during tooth development and ofd1-deficient mice exhibited marked disorganization of molar structure in a previous study (14), suggesting that kif3a may also be associated with tooth development. Dental mesenchymal stem cells, including DFCs and DPCs, can differentiate into osteoblasts and be mineralized. Therefore, the present study hypothesized that kif3a may be involved in the mechanism contributing to dental mesenchymal stem cell osteoblastic differentiation.

In the present study, genetic approaches were used to knock down the IFT protein, kif3a, for the suppression of ciliogenesis, in human DFCs and DPCs and to provide insight into whether kif3a and primary cilia have a direct function in regulating the osteoblastic differentiation of hDFCs and hDPCs via the Wnt signaling pathway. The present study may help elucidate the involvement of kif3a and primary cilia in tooth mesenchymal function and development.

\section{Materials and methods}

Cell culture. Normal impacted third molar tooth germs were collected from patients $(n=10 ; 16-18$ years old $)$ undergoing orthodontic treatment in the West China Hospital of Stomatology (Chengdu, China), following the provision of written informed consent. All the experiments were performed in accordance with the ethical protocol approved by the Ethics Committee of Sichuan University (Chengdu, China). The DFCs and DPCs were isolated and cultured, as previously reported $(15,16)$. In brief, the DFCs and DPCs were isolated from the dental follicle and dental pulp, and incubated in $\alpha$-minimum essential medium ( $\alpha$-MEM; Gibco; Thermo Fisher scientific, Inc., Waltham, MA, USA), supplemented with $10 \%$ fetal bovine serum (FBS; Gibco; Thermo Fisher scientific, Inc.), $100 \mathrm{U} / \mathrm{ml}$ penicillin (Sigma-Aldrich, St. Louis, MO, USA) and $100 \mathrm{mg} / \mathrm{ml}$ streptomycin (Sigma-Aldrich) in humid conditions at $37^{\circ} \mathrm{C}$ of $5 \% \mathrm{CO}_{2}$ in air. The medium was renewed every three days.

Cell characteristics. hDFCs and hDPCs were seeded at a density of $3 \times 10^{3}$ into each well of a 24 -well plate overnight, respectively. The cells were then washed with phosphate-buffered saline (PBS) three times, fixed with $4 \%$ cold polyoxymethylene (Sigma-Aldrich) for $15 \mathrm{~min}$ and permeabilized by $0.5 \%$ Triton (Sigma-Aldrich) for $10 \mathrm{~min}$ at room temperature. Following rinsing three times with PBS, the cells were blocked with $1 \%$ bovine serum albumin (Shanghai Zhangyun Chemical Co., Ltd., Shanghai, China) diluted in PBS for $30 \mathrm{~min}$ at $37^{\circ} \mathrm{C}$. The cells were then treated with primary antibodies for $2 \mathrm{~h}$ in a humidified environment at $37^{\circ} \mathrm{C}$. Following being washed three times with PBS, the cells were treated with secondary antibodies for $1 \mathrm{~h}$ in a humidified environment at $37^{\circ} \mathrm{C}$. Following three rinses with PBS, the nuclei were counterstained with $100 \mathrm{ng} / \mathrm{ml}$ DAPI blue (Beyotime Institute of Biotechnology, Shanghai, China) for $3 \mathrm{~min}$ in the dark. The primary antibodies used were as follows: Anti-vimentin (mouse IgG; 1:200; OMA1-06001; Thermo Fisher Scientific, Inc.), anti-CK14 (mouse IgG;
1:200; MAB3232; EMD Millipore, Billerica, MA, USA) and anti-Stro-1 (monoclonal mouse IgG; 1:100; FAB1038F; R\&D Systems, Minneapolis, MN, USA). Secondary antibodies were conjugated to Alexa FluoR 488 goat anti-mouse (A11001) or Alexa FluoR 555 goat anti-mouse (1:500; A21422; Invitrogen; Thermo Fisher Scientific, Inc.). Images were captured and analyzed under a confocal imaging system (Olympus FV1000; Olympus Corporation, Tokyo, Japan).

Flow-cytometric analysis. To characterize the immunophenotype of the hDFCs and hDPCs, flow cytometric analysis was used to measure the expression of mesenchymal stem cell surface markers. Cells were trypsinized (Hyclone; GE Healthcare Life Sciences, Logan, UT, USA) and incubated at $37^{\circ} \mathrm{C}$ with phycoerthyn (PE)-conjugated cluster of differentiation (CD)29 integrin $\beta 1$ (555443); fluorescein isothiocyanate (FITC)-conjugated CD31 (555445), which is also known as platelet-endothelial cell adhesion molecule-1; FITC-conjugated CD34 (555821), which is also known as hematopoietic progenitor cell antigen; FITC-conjugated CD44 (555478), which is also known as hyaluronate/lymphocyte homing associated cell adhesion molecule; PE-conjugated CD90 (Thy-1/Thy-1.1) (555596); and PE-conjugated CD166 (559263), which is also known as activated leucocyte cell adhesion molecule and is a mesenchymal stem cell marker. All antibodies were purchased from BD Biosciences (Franklin Lakes, NJ, USA). Flow cytometry was performed using a Beckman Coulter Cytomics FC 500 MPL system (Beckman Coulter, Fullerton, CA, USA).

Lentiviral infection. According to previous studies, a total of $5 \times 10^{4} \mathrm{hDFCs}$ and hDPCs were seeded into each well of a 6-well plate at $37^{\circ} \mathrm{C}$ overnight, respectively. At $50 \%$ cell density, $1 \mathrm{ml} \alpha$-MEM (without FBS), containing $1.5 \mu \mathrm{l}$ of green-fluorescence protein-labeled kif3a short hairpin (sh) RNA lentivirus (NeuronBiotech Co., Ltd., Shanghai, China) and $0.5 \mu \mathrm{l}$ polybrene (Sigma-Aldrich), were added into each well. Following incubation for $6 \mathrm{~h}$ at $37^{\circ} \mathrm{C}$, the supernatant was removed and replaced with $2 \mathrm{ml}$ fresh $\alpha$-MEM supplemented with $10 \%$ FBS. After 3 days at $37^{\circ} \mathrm{C}$, the infected cells were observed and images were captured under a fluorescence microscope (Leica Optical, Leica Microsystems GmbH, Wetzlar, Germany) (17).

Osteoblastic induction. For osteoblastic induction, a total of $1 \times 10^{5} \mathrm{hDFCs}$ and hDPCs at passage three were seeded into each well of a 6 -well plate overnight respectively. These cells were then cultured at $37^{\circ} \mathrm{C}$ in osteoblastic inductive medium ( $\alpha$-MEM supplemented with $10 \% \mathrm{FBS}, 10 \mathrm{mmol} / \mathrm{l}$ $\beta$-glycerophosphate (Sigma-Aldrich), $0.2 \mathrm{mmol} / 1$ ascorbic acid (Sigma-Aldrich), $10 \mathrm{nmol} / 1$ 1,25-dihydroxyvitamin D3 and $100 \mathrm{nmol} / \mathrm{l}$ dexamethasone (Sigma-Aldrich) for 3-30 days, depending on the subsequent experiments. The inductive medium was replaced every three days.

Alizarin Red S staining. As previously described (18), following fixation with 4\% paraformaldehyde (Sigma-Aldrich) for $30 \mathrm{~min}$ at room temperature, the cultures were incubated in $0.1 \%$ Alizarin Red solution (Sigma-Aldrich) in Tris $\mathrm{HCl}$ (pH 8.3; 0.5-1 ml per well; Kelong Chemical Co., 
Table I. Oligo-nucleotide primer sequences used in reverse transcription-quantitative polymerase chain reaction analysis.

\begin{tabular}{|c|c|c|}
\hline Gene & Primer sequence $\left(5^{\prime}-3^{\prime}\right)$ & GenBank number \\
\hline ALP & $\begin{array}{l}\text { F: TAAGGACATCGCCTACCAGCTC } \\
\text { R: TCTTCCAGGTGTCAACGAGGT }\end{array}$ & NM_000478.4 \\
\hline DMP1 & $\begin{array}{l}\text { F: GTGAGTGAGTCCAGGGGAGATAA } \\
\text { R: TTTTGAGTGGGAGAGTGTGTGC }\end{array}$ & NM_004407.3 \\
\hline DSPP & $\begin{array}{l}\text { F: CTGTTGGGAAGAGCCAAGATAAG } \\
\text { R: CCAAGATCATTCCATGTTGTCCT }\end{array}$ & NM_014208.3 \\
\hline $\mathrm{OCN}$ & $\begin{array}{l}\text { F: CTCACACTCCTCGCCCTATTG } \\
\text { R: CTCCCAGCCATTGATACAGGTAG }\end{array}$ & NM_199173.3 \\
\hline OPN & $\begin{array}{l}\text { F: CAGTTGTCCCCACAGTAGACAC } \\
\text { R: GTGATGTCCTCGTCTGTAGCATC }\end{array}$ & AB469789.1 \\
\hline Runx2 & $\begin{array}{l}\text { F: CTTTACTTACACCCCGCCAGTC } \\
\text { R: AGAGATATGGAGTGCTGCTGGTC }\end{array}$ & NM_001024630.3 \\
\hline Axin 2 & $\begin{array}{l}\text { F: GACAGGAATCATTCGGCCAC } \\
\text { R: CCTTCAGCATCCTCCGGTAT }\end{array}$ & NM_004655 \\
\hline$\beta$-catenin & $\begin{array}{l}\text { F: CTTACACCCACCATCCCACT } \\
\text { R: CCTCCACAAATTGCTGCTGT }\end{array}$ & NM_001098209 \\
\hline Lef1 & $\begin{array}{l}\text { F: ACAGATCACCCCACCTCTTG } \\
\text { R: ATAGCTGGATGAGGGATGCC }\end{array}$ & NM_016269.1 \\
\hline GAPDH & $\begin{array}{l}\text { F: CTTTGGTATCGTGGAAGGACTC } \\
\text { R: GTAGAGGCAGGGATGATGTTCT }\end{array}$ & NM_002046.3 \\
\hline
\end{tabular}

ALP, alkaline phosphatase; DMP1, dentin matrix protein 1; DSPP, dentin sialophosphoprotein; OCN, osteocalcin; OPN, osteopontin; Runx2, Runt-related transcription factor 2; Axin2, axis inhibition protein 2; Lef1, lymphoid enhancer-binding factor 1; GAPDH, D-glyceraldehyde-3-phosphate; F, forward; R, reverse.

Ltd., Chengdu, China) in a 6 -well plate for $30 \mathrm{~min}$ at room temperature.

Cellular immunofluorescent analysis. The control and kif3a-knockdown hDFCs and hDPCs were seeded $\left(3 \times 10^{3}\right)$ into each well of a 24-well plate overnight, respectively. The methods used were the same as described above. The primary antibodies used were anti-acetylized $\alpha$-tubulin (mouse IgG; 1:500; ab24610; Abcam). Secondary antibodies were conjugated to Alexa FluoR 555 (goat anti-mouse; 1:500; Invitrogen; Thermo Fisher Scientific, Inc.). Images were captured and analyzed under a confocal imaging system (Olympus FV1000; Olympus Corporation).

$R N A$ preparation and reverse transcription-quantitative polymerase chain reaction $(R T-q P C R)$ analysis. The control and kif3a-knockdown hDFCs and hDPCs were harvested from each well of a 6 -well plate following 3 days osteoblastic induction in preparation for RNA isolation. Total RNA was obtained using RNA iso ${ }^{\text {TM }}$ Plus (Takara Bio Inc., Otsu, Japan). cDNAs were synthesized by reverse transcription using the extracted RNA with PrimeScript ${ }^{\circledR}$ Perfect Real Time reagent kit (Takara Bio., Inc.). The relative expression levels of genes were quantified using qPCR with SYBR ${ }^{\circledR}$ Premix Ex Taq $^{\mathrm{TM}}$ (Perfect Real Time; Takara Bio, Inc.) in a $10 \mu \mathrm{l}$ total reaction volume using an ABI Prism 7300 system (Applied
Biosystems; Thermo Fisher Scientific, Inc.). The qPCR conditions were as follows: Initial cycle was $95^{\circ} \mathrm{C}$ for $30 \mathrm{sec}$, 40 cycles at $95^{\circ} \mathrm{C}$ for $5 \mathrm{sec}$ and $60^{\circ} \mathrm{C}$ for $30 \mathrm{sec}$; the final cycle was $95^{\circ} \mathrm{C}$ for $15 \mathrm{sec}, 60^{\circ} \mathrm{C}$ for $1 \mathrm{~min}$ and $95^{\circ} \mathrm{C}$ for $15 \mathrm{sec}$. Dissociation curves were used to confirm primers specificity. D-glyceraldehyde-3-phosphate (GAPDH) was used as an internal reference, and relative mRNA levels were quantified using the $2^{-\Delta \Delta \mathrm{Cq}}$ method (19). The primer sequences used in the present study for GAPDH, dentin sialophosphoprotein (DSPP), dentin matrix protein 1 (DMP-1), osteocalcin (OCN), Runt-related transcription factor 2 (Runx2), alkaline phosphatase (ALP), osteopontin (OPN), axis inhibition protein 2 (Axin2) and $\beta$-catenin, lymphoid enhancer-binding factor 1 (Lef1) are listed in Table I. All experiments were performed three times.

Western blot analysis. Control and kif3a-knockdown hDFCs and hDPCs were harvested from each well of a 6-well plate following 1 week of osteoblastic induction prior to protein extraction. Cells were rinsed three times in PBS prior to being lysed using cell lysis buffer, containing $0.5 \%$ $100 \mathrm{mmol} / 1$ phenylmethanesulfonyl fluoride, $0.1 \%$ protease inhibitor and $0.5 \%$ phosphatase inhibitor, on ice. Proteins in the supernatant were measured by modified Bradford Protein Assay (Bio-Rad Laboratories, Inc., Hercules, CA, USA). An equal quantity of total protein $(50 \mathrm{mg})$ was separated by 


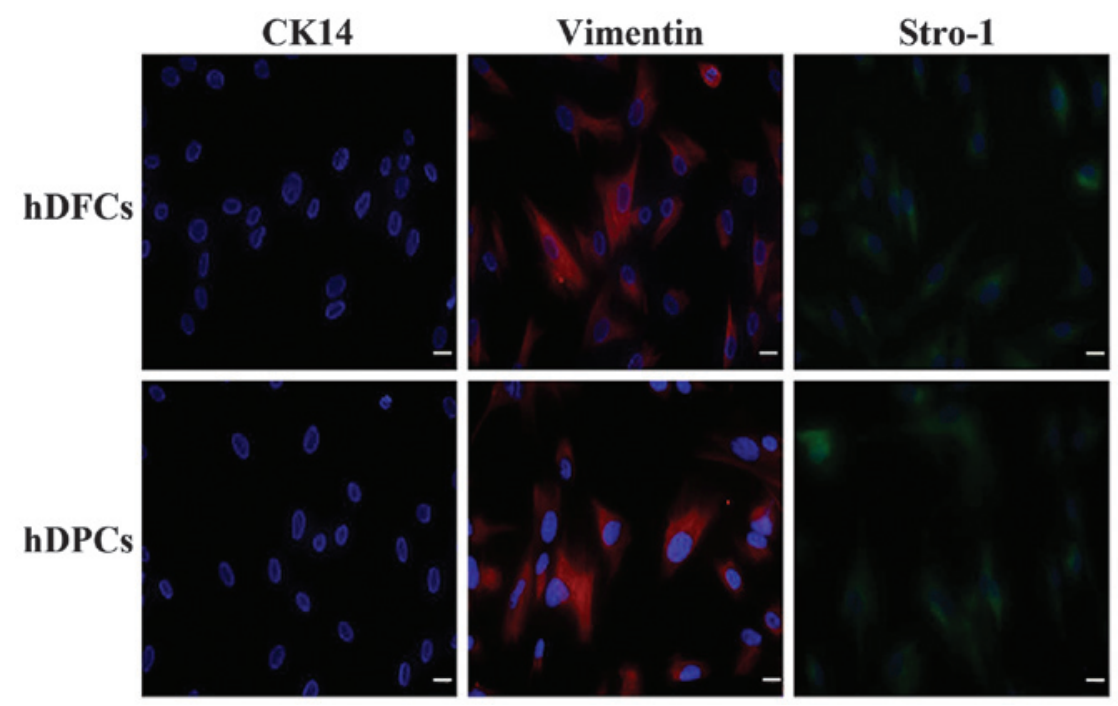

Figure 1. Evaluation of the biological characteristics of hDFCs and hDPCs. The two types of cell were positive for vimentin and Stro-1, but negative for the epithelial marker, CK-14. Scale bar=20 $\mu \mathrm{m}$. hDFCs, human dental follicle cells; hDPCs, human dental pulp cells.

sodium dodecyl sulfate polyacrylamide gel electrophoresis (SDS-PAGE) and transferred onto polyvinylidene fluoride membranes (Invitrogen; Thermo Fisher Scientific, Inc.). Following blocking with non-fat milk in PBS with Tween 20 (PBST) for $1 \mathrm{~h}$ at room temperature, the membranes were incubated with primary antibodies at $4^{\circ} \mathrm{C}$ overnight. The membranes were subsequently washed three times with PBST for $10 \mathrm{~min}$ and incubated with secondary antibodies at room temperature for $1 \mathrm{~h}$, respectively. Membranes were visualized with a chemiluminescent horseradish peroxidase (HRP) substrate (EMD Millipore) using a ChemiDoc XRS system (Bio-Rad Laboratories, Inc.). The primary antibodies used for western blot analysis were as follows: Anti- $\beta$-actin (1:500; ab8227), anti-ALP (1:500; ab67228), anti-DMP1 (1:500; ab103203), anti-kif3a (1:500; ab133587), anti-bone sialoprotein (BSP; 1:200; ab52128), anti-OPN (1:1,000; ab8448), anti-Runx2 (1:500; ab76956), anti-phosphorylated glycogen synthase kinase $3 \beta$ (GSK3 $\beta ; 1: 1,000$; ab32391; Abcam), anti-Lef1 (1:500; ab85052) and anti-active $\beta$-catenin (1:4,000; 8814; Cell Signaling Technology, Inc., Danvers, MA, USA). The secondary antibodies were HRP-conjugated AffiniPure goat anti-mouse IgG (H+L; 1:10,000; ZB-2305) and HRP-conjugated AffiniPure goat anti-rabbit $\mathrm{IgG}(\mathrm{H}+\mathrm{L}$; 1:10,000, ZB-2301; ZSGB-Bio, Beijing, China). All experiments were performed three times.

Statistical analysis. The data in the present study were collected from three different samples of the same cell sample in triplicate. Values are presented as the mean \pm standard deviation. Statistical significance was evaluated using one-way analysis of variance using SPSS software (Version 10.0; SPSS, Inc., Chicago, IL, USA). P $<0.05$ was considered to indicate a statistically significant difference.

\section{Results}

Characterization of hDFCs and hDPCs as dental mesenchymal stem/precursor cells. The present study identified the characterization of hDFCs and hDPCs as dental mesenchymal stem cells using cellular immunofluorescent detection. The two cell types were positive for the vimentin and Stro-1 mesenchymal stem cells markers, but negative for the CK-14 epithelial cell marker (Fig. 1). The immunophenotypic characterization was performed using flow cytometry. The DFCs and DPCs were positive for the CD29, CD44, CD90 and CD166 mesenchymal stem cell markers. However, the two types of cell were negative for the leucocyte precursor markers, CD31 and CD34, suggesting a lack of cells of hematopoietic and angiogenic lineages (Fig. 2). These data indicated that the DPC and DFC cell lineage were pure mesenchymal cells and maintained stem cell-like properties.

Knockdown of kif3a leads to loss of primary cilia in hDFCs and hDPCs. The present study then investigated the existence of primary cilia in the hDFCs and hDPCs. The primary cilia were immunostained using acetylated $\alpha$-tubulin antibody and observed under a laser scanning confocal microscope, as described previously (14) (Fig. 3A). The expression of kif3a was then determined using western blot analysis, and the target bands were detected with purified antipeptide antibodies in the two cell cultures (Fig. 3B) Lentiviruses expressing four different shRNAs specific for kif3a were transfected into the cells, as described above, and the shRNA with the most marked effect was selected. The efficiency of kif3a suppression was determined using immunoblot analysis (Fig. 3C). Following kif3a knockdown, the number of primary cilia were substantially reduced in the two cell cultures (Fig. 3D-G).

Knockdown of kif3a suppresses osteoblastic differentiation in hDFCs and hDPCs. To examine the association between the loss of primary cilia caused by kif3a knockdown and osteoblastic differentiation in hDFCs and hDPCs, the present study performed Alizarin Red staining following treatment with osteoblastic inductive medium. Following 10 days induction, the kif3a-knockdown cells formed fewer mineralized nodules, compared with the cells in the control group, in the hDFCs and hDPCs. This difference became more significant following 30 days induction (Fig. 4A and B). 

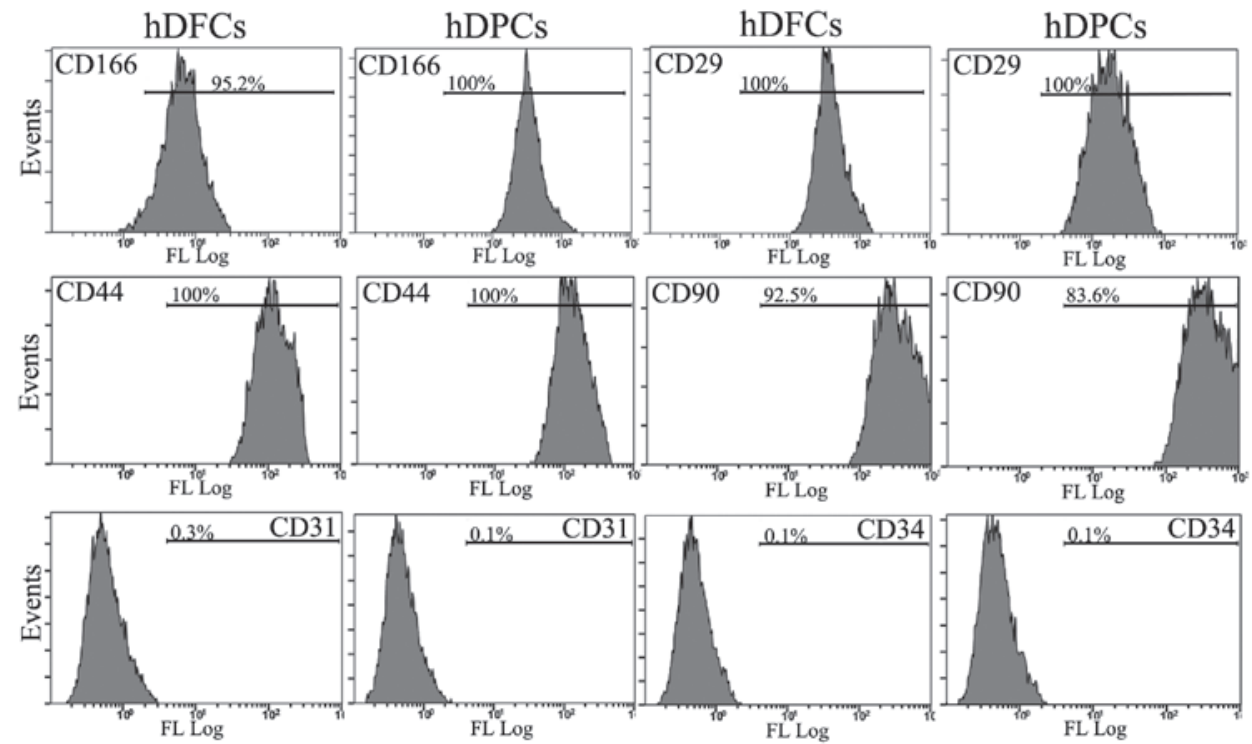

Figure 2. Analysis of cell surface antigens in hDFCs and hDPCs. Flow cytometric analysis indicated that the hDFCs and hDPCs cells were of mesenchymal origin (positive for CD29, CD44 and CD90, and negative for CD31 and CD34) and stem cells (positive for CD166). hDFCs, human dental follicle cells; hDPCs, human dental pulp cells.
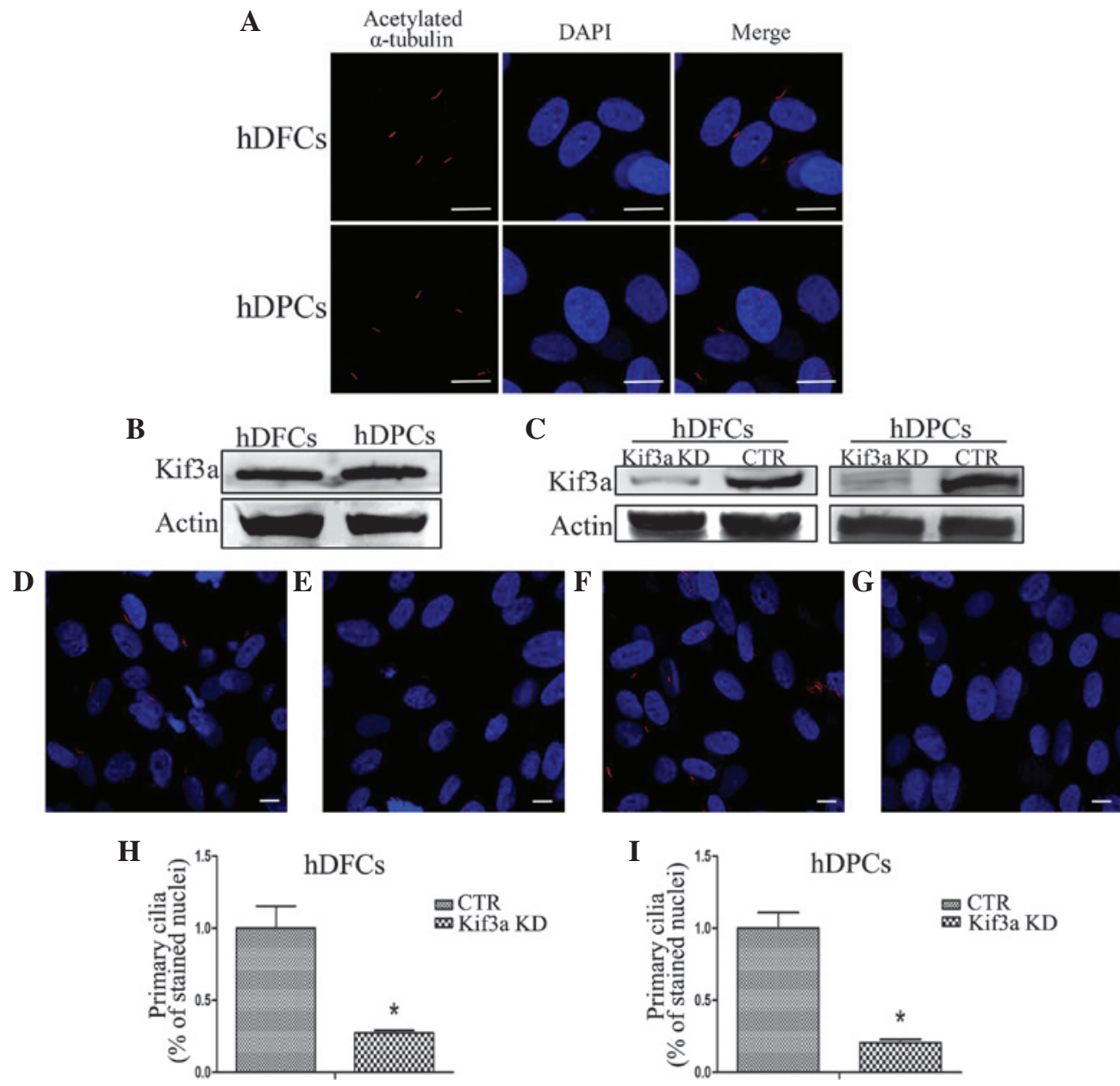

Figure 3. Evidence for expression of kif3a and primary cilia in hDPCs and hDFCs. (A) Immunofluorescence analysis of primary cilia in hDFCs and hDPCs. Immunostaining of primary cilia was performed with acetylated $\alpha$-tubulin antibody (red dots) and counterstaining with DAPI. (B) Western blot showing the expression of kif3a in hDFCs and hDPCs. (C) Expression of kif3a was notably reduced following knockdown in the hDFCs and hDPCs. (D-G) A marked reduction in cilia numbers were observed in the (E and G) kif3a-knockdown hDFCs and hDPCs, compared with the (D and F) control hDFCs and hDPCs, visualized using a laser scanning confocal microscope. Immunostaining of primary cilia (red dots) was performed with acetylated $\alpha$-tubulin antibody. Counterstaining with DAPI was used to calculate the percentage of primary cilia in the hDFCs and hDPCs. (H and I) Statistical analyses of the percentage of primary cilia. Data are presented as the mean \pm standard deviation. ${ }^{*} \mathrm{P}<0.05$, vs. control. Scale bar $=10 \mu \mathrm{m}$. hDFCs, human dental follicle cells; hDPCs, human dental pulp cells; CTR, control; kif3a KD, kif3a knockdown. 

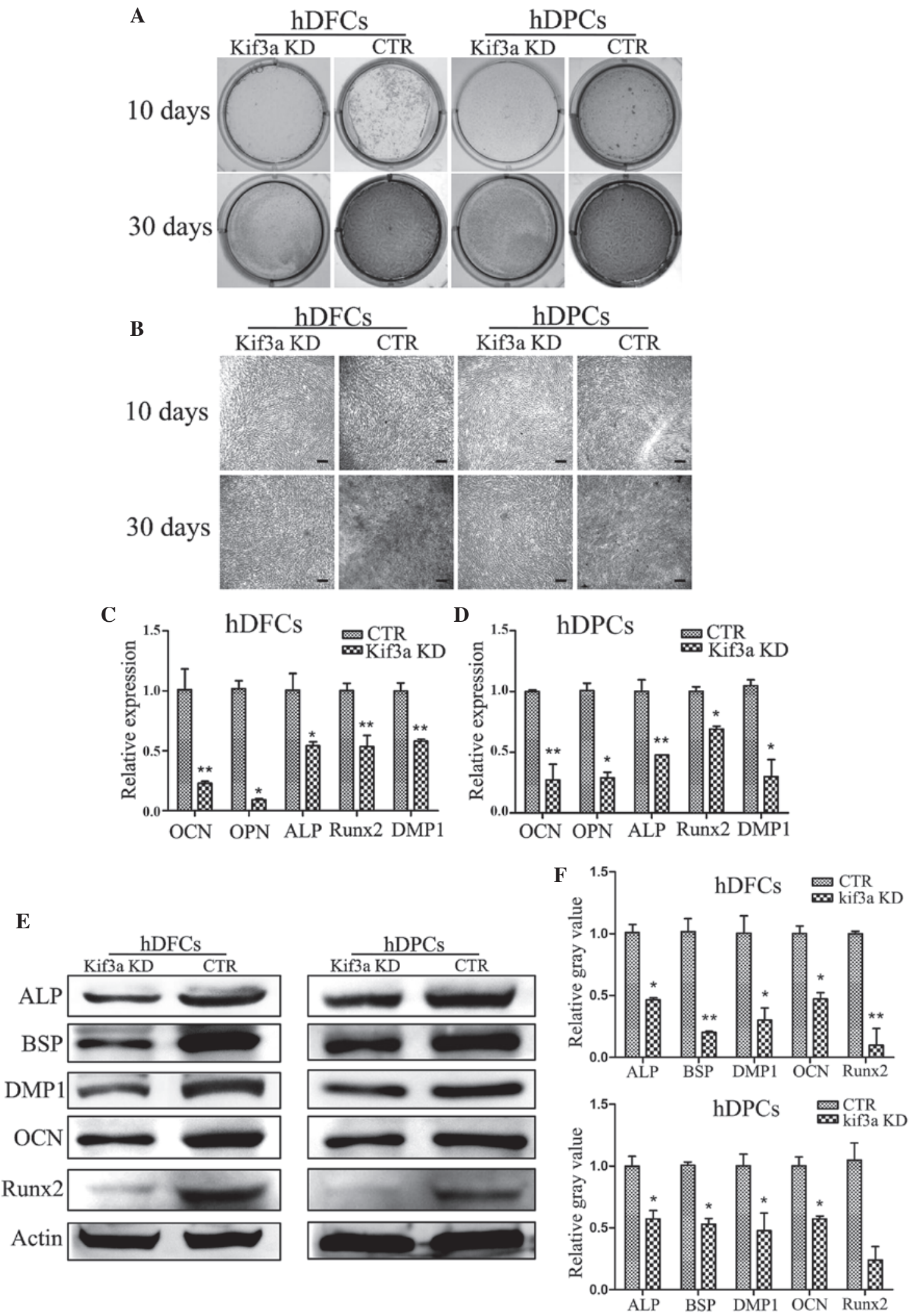

Figure 4. Effects of kif3a knockdown on osteoblastic differentiation in hDFCs and hDPCs. (A and B) Alizarin Red staining for 10 and 30 days. (B) Staining viewed under magnification (Scale bar $=400 \mu \mathrm{m}$ ). Results of (C and D) reverse transcription-quantitative polymerase chain reaction and (E and F) western blot analyses following 6 days induction. Data are representative of three independent experiments and are presented as the mean \pm standard deviation. ${ }^{*}<0.05$ and ${ }^{* *} \mathrm{P}<0.01$ vs. control. hDFCs, human dental follicle cells; hDPCs, human dental pulp cells; CTR, control; kif3a KD, kif3a-knockdown; ALP, alkaline phosphatase; DMP1, dentin matrix protein 1; BSP, bone sialoprotein; OCN, osteocalcin; OPN, osteopontin; Runx2, Runt-related transcription factor 2.

The present study then assessed the expression levels of osteoblastic genes using RT-qPCR (Fig. 4C and D), and protein expression levels using western blot analysis (Fig. 4E). The results showed that the osteoblastic genes and 

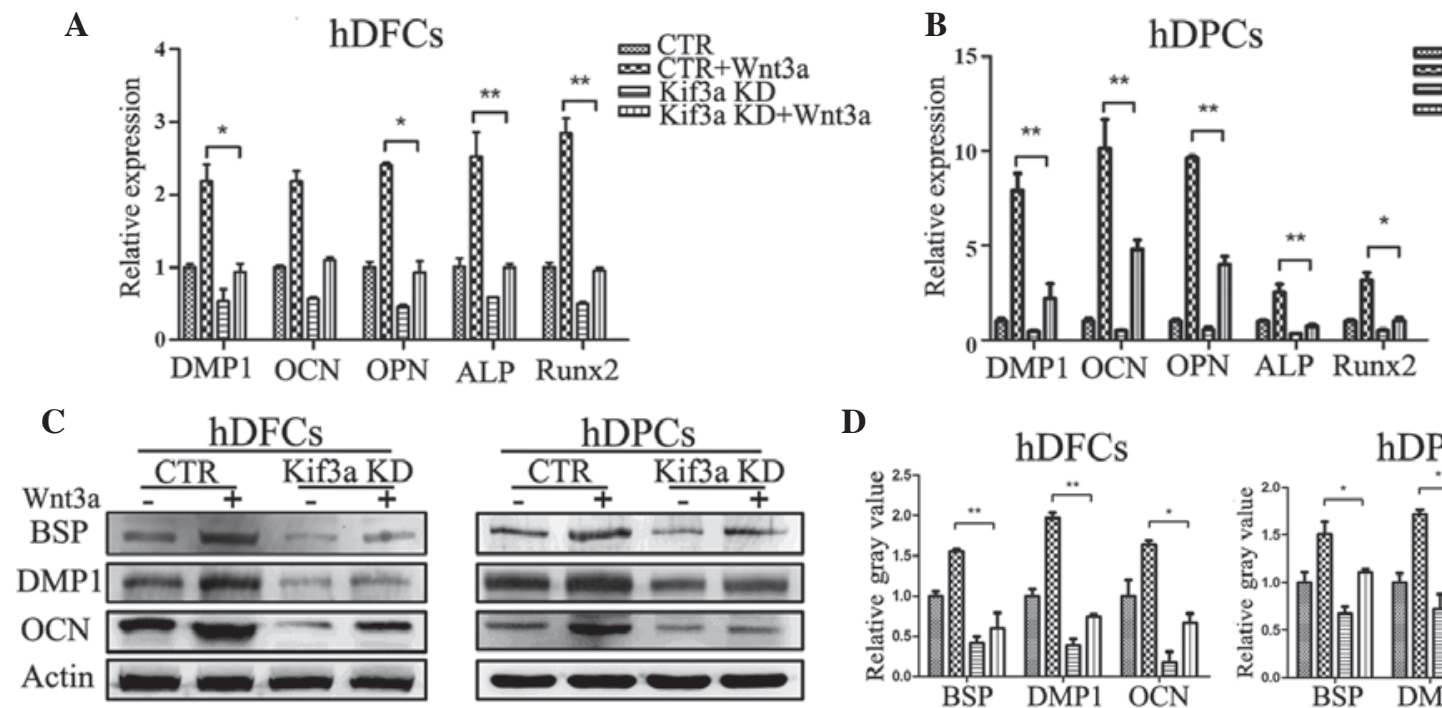

D
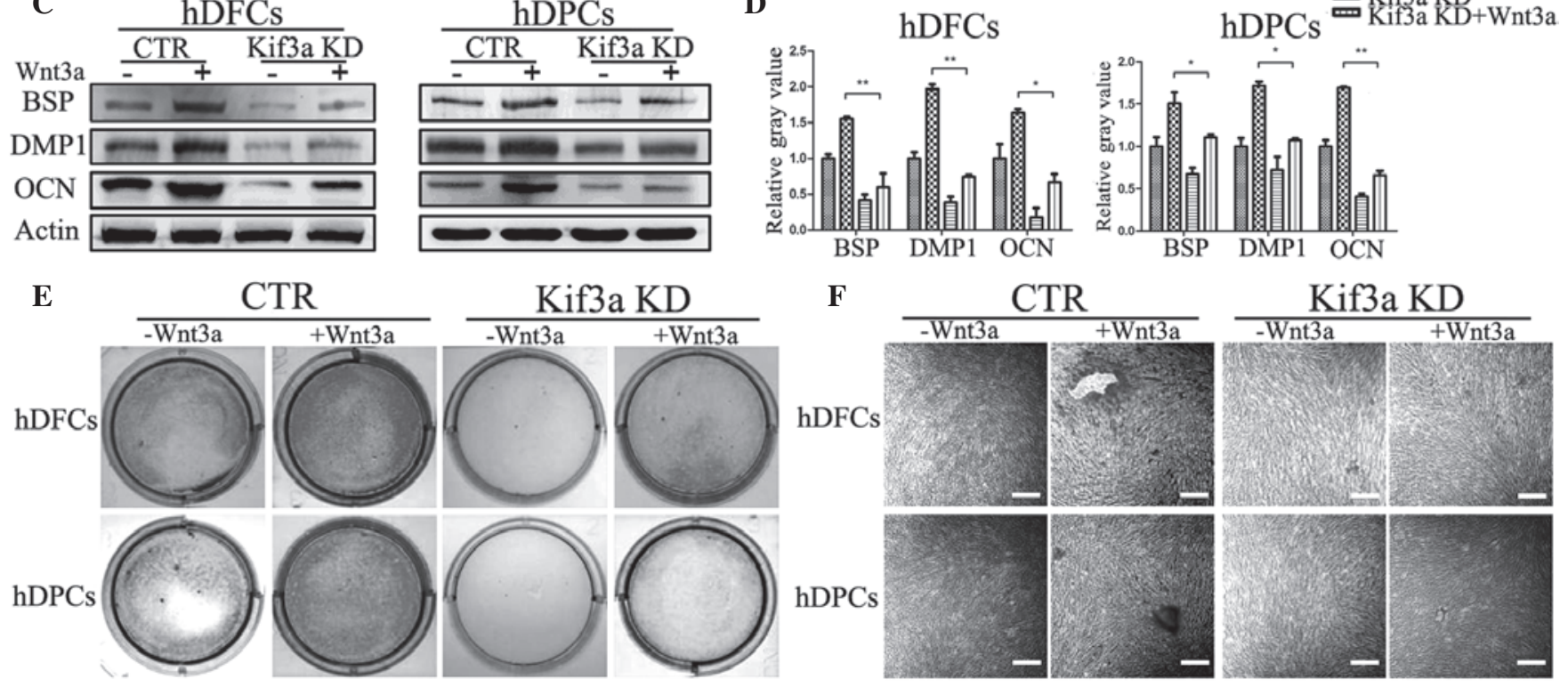

Figure 5. Osteoblastic differentiation of hDFCs and hDPCs is rescued by exogenous Wnt3a. Effects on the mRNA levels of osteoblastic markers of (A) hDPCs and (B) hDFCs following stimulation with $100 \mathrm{ng} / \mathrm{ml}$ recombinant Wnt3a for $18 \mathrm{~h}$ were assessed using reverse transcription-quantitative polymerase chain reaction analysis. Data are presented as the mean \pm standard deviation. (C) Effects on protein levels were examined following 6 days stimulation using Western blot analysis and were subsequently (D) quantified. (E) After 14 days of treatment, culture plates were analyzed for mineralization using Alizarin Red staining, and (F) visualized at magnification (scale bar=200 $\mu \mathrm{m}$ ). $\mathrm{P}<0.05 ;{ }^{* *} \mathrm{P}<0.01$. hDFCs, human dental follicle cells; hDPCs, human dental pulp cells; CTR, control; kif3a KD, kif3a knockdown; ALP, alkaline phosphatase; DMP1, dentin matrix protein 1; BSP, bone sialoprotein; OCN, osteocalcin; OPN, osteopontin; Runx2, Runt-related transcription factor 2 .

proteins, including ALP, BSP, DMP1, OCN and Runx2, were significantly downregulated following kif3a knockdown in the hDFC and hDPC cells.

Exogenous Wnt3a partially recovers osteoblastic differentiation in hDFCs and hDPCs. To examine the mechanisms by which the loss of kif3a causes alterations in hDFC and hDPC functions, the present study examined whether the Wnt signaling pathway was involved in this suppression, which has a close association with tooth development $(20,21)$. Following treatment with osteoblastic medium and recombinant Wnt3a for 6 days, the mRNA and protein expression levels of osteoblastic markers of hDPCs and hDFCs, were assessed, respectively. Significant increases in the mRNA (Fig. 5A and B) and protein (Fig. 5C) expression levels of osteoblastic markers were found in the Wnt3a-treated wild-type cells, compared with the control cells. By contrast, there was no significant increase in the kif3a-knockdown cells (Fig. 5A-C). In accordance with these results, the kif3a-knockdown cells formed fewer mineralized nodules, compared with the control group cells, in the hDFCs and hDPCs following culture in osteoblastic medium for 14 days and treatment with recombinant Wnt3a (Fig. 5D and E). This confirmed that the loss of kif3a suppressed osteoblastic differentiation in the hDFCs and hDPCs by the inhibition of Wnt signaling.

Loss of kif3a causes abnormal Wnt signaling in hDFCs and $h D P C s$. The present study further examined whether the loss of primary cilia of the hDFCs and hDPCs following inhibition of kif3a leads to interference in the canonical Wnt pathway. Using RT-qPCR and western blot analyses, it was found that the expression levels of canonical Wnt pathway factors, including Axin2, $\beta$-catenin and Lef1, were significantly lower in the kif3a-knockdown cells, compared with the control cells, at the transcriptional (Fig. 6A and B) and translational (Fig. 6C) levels following treatment in osteoblastic medium for 6 days. However, the addition of $100 \mathrm{ng} / \mathrm{ml}$ recombinant human Wnt3a protein to the osteoblastic hDFC and hDPC cultures resulted in a significant increase in Wnt signaling activity, at the mRNA (Fig. 6D and E) and protein (Fig. 6F) expression levels, in the control cells. By contrast, there was no significant increase in the kif3a-knockdown cells (Fig. 6D-F), suggesting that the loss of kif3a impaired the Wnt signaling response in the hDFCs and hDPCs. This result confirmed that kif3a, at least part, regulated $\mathrm{hDFC}$ and hDPC osteoblastic differentiation by the Wnt signaling pathway. 
A

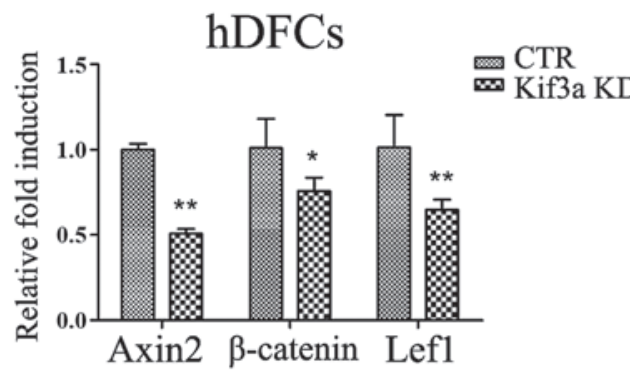

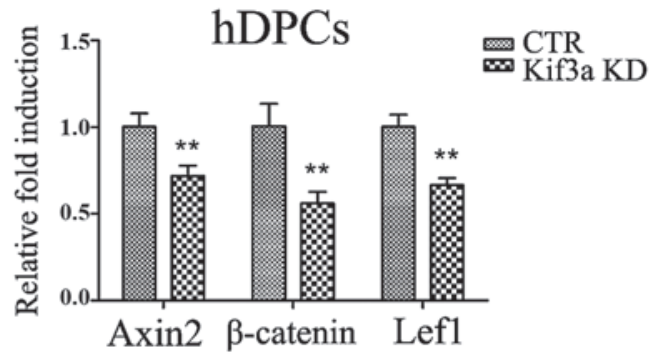

C

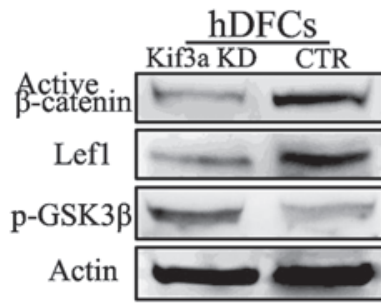

$\mathrm{hDPCs}$

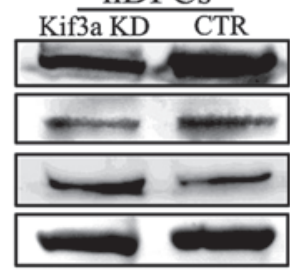

D

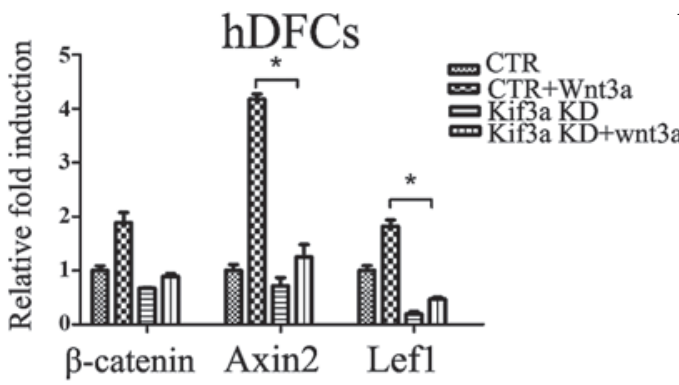

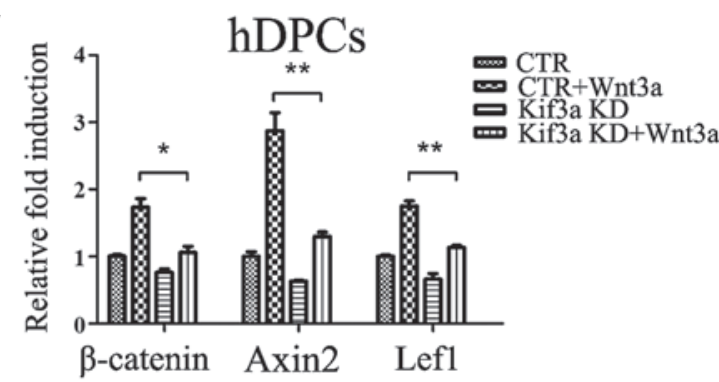

$\mathbf{F}$
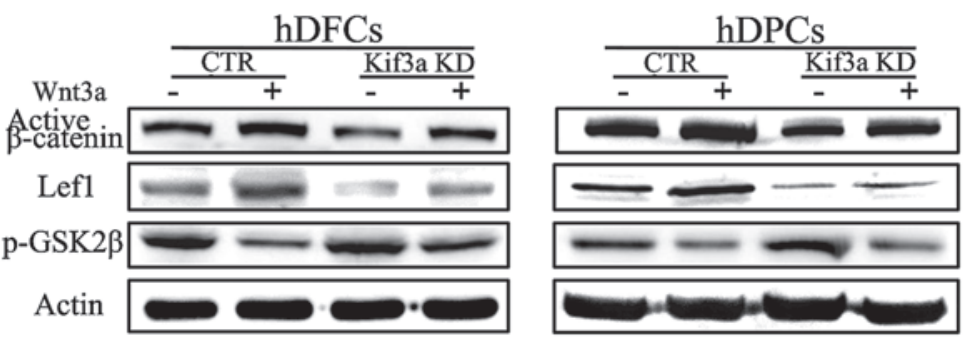

Figure 6. Effects of kif3a knockdown on Wnt signaling in hDFCs and hDPCs. There was a decrease of Wnt signaling on (A and B) gene expression levels, revealed using reverse transcription-quantitative polymerase chain reaction analysis, and on (C) protein levels, revealed using Western blot analysis following 6 days osteoblastic induction in hDFCs and hDPCs. (D and E) mRNA and (F) protein expression levels of Wnt signaling molecules were markedly higher in the control cells, compared with the kif3a-knockdown cells following $100 \mathrm{ng} / \mathrm{ml}$ recombinant Wnt $3 \mathrm{a}$ stimulation for $18 \mathrm{~h}$. Data are presented as the mean \pm standard deviation. " $\mathrm{P}<0.05$ and ${ }^{* *} \mathrm{P}<0.001$, vs. control. hDFCs, human dental follicle cells; hDPCs, human dental pulp cells; CTR, control; kif3a KD, kif3a knockdown; Axin2, axis inhibition protein 2; Lef1, lymphoid enhancer-binding factor 1; p-GSK3 $\beta$, phosphorylated glycogen synthase kinase $3 \beta$.

\section{Discussion}

The kif3a protein is involved in embryogenesis through a ciliary mechanism, which was initially reported in mice with kif3a deficiency lacking cilia and exhibiting numerous structural abnormalities (22). In several stem/progenitor cell lines, kif3a is also reported to be involved in the cell differentiation (23-25). The present study showed that two dental-derived mesenchymal stem/precursor cells, hDFCs and hDPCs, are cells with primary cilia, rather than just odontoblasts, as described in previous reports $(14,26)$. The knockdown of kif3a in the hDFCs and hDPCs resulted in a reduction in the numbers of primary cilia, consistent with the known role of kif3a in primary cilia formation $(27,28)$.
Wnt signaling has been shown to be an important regulatory pathway in the osteogenic differentiation of mesenchymal stem cells (29-31). The persistent expression of $\beta$-catenin in the dental mesenchyme results in the premature differentiation of odontoblasts and differentiation of cementoblasts, and induces immoderate dentin and cementum generation in vivo (32). In several tissues, kif3a has been reported to be involved in regulating the Wnt pathway as an IFT motor subunit of primary cilia $(11,33,34)$.

The present study demonstrated that kif3a-knockdown hDFCs and hDPCs caused abnormality of the Wnt signal pathway. Lef1 and active $\beta$-catenin were significantly downregulated following kif3a knockdown. kif3a knockdown also attenuated the exogenous Wnt3a-stimulated expression levels 
of Lef1 and active $\beta$-catenin in the hDFCs and hDPCs (Fig. 6). In the present study, the addition of recombinant Wnt3a protein upregulated osteoblastic gene and protein expression levels, and formed more mineralized nodules in vitro. This Wnt3a-induced expression of osteoblastic markers was markedly reduced in the kif3a-knockdown cells, in accordance with the effect of kif3a on the Wnt signaling pathway, which indicated the importance of kif3a and the integrity of primary cilia in the differentiation in hDFCs and hDPCs in a Wntdependent manner.

Several lines of evidence support the hypothesis that cilia and cilia-associated proteins regulate Wnt signaling in a varied manner $(11,33,35-37)$. It is possible that the role of cilia and cilia-associated proteins in the Wnt pathway is tissue or stage-specific. With bone mesenchymal cell differentiation as an example, Chen et al (38) and Silkstone et al (39) found that $\beta$-catenin signaling activation inhibited the differentiation of mesenchymal stem cells (38). However, in cells committed to the osteoblast lineage, osteoblast differentiation was enhanced by $\beta$-catenin (39). In the present study, the disruption of kif3a led to a reduction of Wnt signaling, consistent with the role of kif3a in osteoblasts and prostate cancer cells demonstrated in previous reports $(11,33,40)$. Further investigations are required to confirm the role of kif3a in regulating cilia-dependent Wnt signaling in tooth development and repair. Although further elucidation is required, analysis of the data obtained in the present study demonstrated that kif3a was essential in hDFC and hDPC differentiation though Wnt signaling.

In conclusion, the present study provided novel insight into the mechanisms of primary cilia as an indispensable organelle regulating the osteoblastic differentiation of hDFCs and hDPCs. The results suggested that the cilia-associated kinesin protein, kif3a, may be a regulator of hDFC and hDPC osteoblastic differentiation via the Wnt signaling pathway, and offer potential as a therapeutic target in diseases associated with cilia. These results not only enhance current understanding of tooth development and diseases of tooth mineralization, but also indicate possible strategies to regulate mineralization during tooth repair and regeneration.

\section{Acknowledgements}

The present study was supported by the National Natural Science Foundation of China (grant no. 81271119) and the Basic Research Program of the Sichuan Province of China (grant no. 2013JY0019).

\section{References}

1. Yang B, Chen G, Li J, Zou Q, Xie D, Chen Y, Wang H, Zheng X, Long $\mathrm{J}$ and Tang $\mathrm{W}$ : Tooth root regeneration using dental follicle cell sheets in combination with a dentin matrix-based scaffold. Biomaterials 33: 2449-2461, 2012.

2. Nie X, Tian W, Zhang Y, Chen X, Dong R, Jiang M, Chen F and Jin Y: Induction of transforming growth factor-beta 1 on dentine pulp cells in different culture patterns. Cell Biol Int 30: 295-300, 2006.

3. Jiao L, Xie L, Yang B, Yu M, Jiang Z, Feng L, Guo W and Tian W: Cryopreserved dentin matrix as a scaffold material for dentin-pulp tissue regeneration. Biomaterials 35: 4929-4939, 2014.

4. Yang C, Sun L, Li X, Xie L, Yu M, Feng L, Jiang Z, Guo W and Tian W: The potential of dental stem cells differentiating into neurogenic cell lineage after cultivation in different modes in vitro. Cell Reprogram 16: 379-391, 2014.
5. Wang X, He F, Tan Y, Tian W and Qiu S: Inhibition of Delta1 promotes differentiation of odontoblasts and inhibits proliferation of human dental pulp stem cell in vitro. Arch Oral Biol 56: 837-845, 2011.

6. Guo L, Li J, Qiao X, Yu M, Tang W, Wang H, Guo W and Tian W: Comparison of odontogenic differentiation of human dental follicle cells and human dental papilla cells. PLoS One 8: e62332, 2013.

7. Kolpakova-Hart E, Jinnin M, Hou B, Fukai N and Olsen BR: Kinesin-2 controls development and patterning of the vertebrate skeleton by Hedgehog- and Gli3-dependent mechanisms. Dev Biol 309: 273-284, 2007.

8. Rosenbaum JL and Witman GB: Intraflagellar transport. Nat Rev Mol Cell Biol 3: 813-825, 2002.

9. Tong CK, Han YG, Shah JK, Obernier K, Guinto CD and Alvarez-Buylla A: Primary cilia are required in a unique subpopulation of neural progenitors. Proc Natl Acad Sci USA 111: 12438-12443, 2014.

10. D'Amico E, Gayral S, Massart C, Van Sande J, Reiter JF, Dumont JE, Robaye B and Schurmans S: Thyroid-specific inactivation of KIF3A alters the TSH signaling pathway and leads to hypothyroidism. J Mol Endocrinol 50: 375-387, 2013.

11. Qiu N, Xiao Z, Cao L, Buechel MM, David V, Roan E and Quarles LD: Disruption of Kif3a in osteoblasts results in defective bone formation and osteopenia. J Cell Sci 125: 1945-1957, 2012.

12. Temiyasathit S, Tang WJ, Leucht P, Anderson CT, Monica SD, Castillo AB,Helms JA, Stearns T and Jacobs CR: Mechanosensing by the primary cilium: deletion of Kif3A reduces bone formation due to loading. PLoS One 7: e33368, 2012.

13. Xiao ZS and Quarles LD: Role of the polycytin-primary cilia complex in bone development and mechanosensing. Ann N Y Acad Sci 1192: 410-421, 2010.

14. Thivichon-Prince B, Couble ML, Giamarchi A, Delmas P, Franco B, Romio L, Struys T, Lambrichts I, Ressnikoff D, Magloire H and Bleicher F: Primary cilia of odontoblasts: Possible role in molar morphogenesis. J Dent Res 88: 910-915, 2009.

15. Guo W, Chen L, Gong K, Ding B, Duan Y and Jin Y: Heterogeneous dental follicle cells and the regeneration of complex periodontal tissues. Tissue Eng Part A 18: 459-470, 2012.

16. Morsczeck C, Götz W, Schierholz J, Zeilhofer F, Kühn U, Möhl C, Sippel C and Hoffmann KH: Isolation of precursor cells (PCs) from human dental follicle of wisdom teeth. Matrix Biol 24: 155-165, 2005.

17. Wang X, He H, Wu X, Hu J and Tan Y: Promotion of dentin regeneration via CCN3 modulation on Notch and BMP signaling pathways. Biomaterials 35: 2720-2729, 2014.

18. Yu J, He H, Tang C, Zhang G, Li Y, Wang R, Shi J and Jin Y: Differentiation potential of STRO-1+ dental pulp stem cells changes during cell passaging. BMC Cell Biol 11: 32, 2010.

19. Livak KJ and Schmittgen TD: Analysis of relative gene expression data using real-time quantitative PCR and the 2 (-Delta Delta C (T)) Method. Methods 25: 402-408, 2001.

20. Chen J, Lan Y, Baek JA, Gao Y and Jiang R: Wnt/beta-catenin signaling plays an essential role in activation of odontogenic mesenchyme during early tooth development. Dev Biol 334: 174-185, 2009.

21. Zhu X, Zhao P, Liu Y, Zhang X, Fu J, Ivy Yu HM, Qiu M, Chen Y, Hsu W and Zhang Z: Intra-epithelial requirement of canonical Wnt signaling for tooth morphogenesis. J Biol Chem 288: 12080-12089, 2013.

22. Marszalek JR, Ruiz-Lozano P, Roberts E, Chien KR and Goldstein LS: Situs inversus and embryonic ciliary morphogenesis defects in mouse mutants lacking the KIF3A subunit of kinesin-II. Proc Natl Acad Sci USA 96: 5043-5048, 1999.

23. Guadiana SM, Semple-Rowland S, Daroszewski D, Madorsky I, Breunig JJ, Mykytyn K and Sarkisian MR: Arborization of dendrites by developing neocortical neurons is dependent on primary cilia and type 3 adenylyl cyclase. J Neurosci 33: 2626-2638, 2013.

24. Zhao C, Omori Y, Brodowska K, Kovach P and Malicki J: Kinesin-2 family in vertebrate ciliogenesis. Proc Natl Acad Sci USA 109: 2388-2393, 2012.

25. Zhu D, Shi S, Wang H and Liao K: Growth arrest induces primary-cilium formation and sensitizes IGF-1-receptor signaling during differentiation induction of 3T3-L1 preadipocytes. J Cell Sci 122: 2760-2768, 2009.

26. Couble ML, Farges JC, Bleicher F, Perrat-Mabillon B, Boudeulle M and Magloire H: Odontoblast differentiation of human dental pulp cells in explant cultures. Calcif Tissue Int 66: 129-138, 2000. 
27. Barakat MT, Humke EW and Scott MP: Kif3a is necessary for initiation and maintenance of medulloblastoma. Carcinogenesis 34 : 1382-1392, 2013

28. Lin F, Hiesberger T, Cordes K, Sinclair AM, Goldstein LS, Somlo S and Igarashi P: Kidney-specific inactivation of the KIF3A subunit of kinesin-II inhibits renal ciliogenesis and produces polycystic kidney disease. Proc Natl Acad Sci USA 100: 5286-5291, 2003.

29. Delaine-Smith RM, Sittichokechaiwut A and Reilly GC: Primary cilia respond to fluid shear stress and mediate flow-induced calcium deposition in osteoblasts. FASEB J 28: 430-439, 2014.

30. Holmen SL, Zylstra CR, Mukherjee A, Sigler RE, Faugere MC, Bouxsein ML, Deng L, Clemens TL and Williams BO: Essential role of beta-catenin in postnatal bone acquisition. J Biol Chem 280: 21162-21168, 2005.

31. Leucht P, Monica SD, Temiyasathit S, Lenton K, Manu A, Longaker MT, Jacobs CR, Spilker RL, Guo H, Brunski JB and Helms JA: Primary cilia act as mechanosensors during bone healing around an implant. Med Eng Phys 35: 392-402, 2013

32. Kim TH, Lee JY, Baek JA, Lee JC, Yang X, Taketo MM, Jiang R and Cho ES: Constitutive stabilization of ß-catenin in the dental mesenchyme leads to excessive dentin and cementum formation. Biochem Biophys Res Commun 412: 549-555, 2011.

33. Liu Z, Rebowe RE, Wang Z, Li Y, Wang Z, DePaolo JS, Guo J, Qian C and Liu W: KIF3a promotes proliferation and invasion via Wnt signaling in advanced prostate cancer. Mol Cancer Res 12: 491-503, 2014.
34. Gerdes JM, Liu Y, Zaghloul NA, Leitch CC, Lawson SS, Kato M, Beachy PA, Beales PL, DeMartino GN, Fisher S, et al: Disruption of the basal body compromises proteasomal function and perturbs intracellular Wnt response. Nat Genet 39: 1350-1360, 2007.

35. Han YG, Kim HJ, Dlugosz AA, Ellison DW, Gilbertson RJ and Alvarez-Buylla A: Dual and opposing roles of primary cilia in medulloblastoma development. Nat Med 15: 1062-1065, 2009.

36. Kishimoto N, Cao Y, Park A and Sun Z: Cystic kidney gene seahorse regulates cilia-mediated processes and Wnt pathways. Dev Cell 14: 954-961, 2008.

37. Lancaster MA, Louie CM, Silhavy JL, Sintasath L, Decambre M, Nigam SK, Willert K and Gleeson JG: Impaired Wnt-beta-catenin signaling disrupts adult renal homeostasis and leads to cystic kidney ciliopathy. Nat Med 15: 1046-1054, 2009.

38. Chen Y, Whetstone HC, Lin AC, Nadesan P, Wei Q, Poon R and Alman BA: Beta-catenin signaling plays a disparate role in different phases of fracture repair: Implications for therapy to improve bone healing. PLoS Med 4: e249, 2007.

39. Silkstone D, Hong $\mathrm{H}$ and Alman BA: Beta-catenin in the race to fracture repair: In it to Wnt. Nat Clin Pract Rheumatol 4: 413-419, 2008

40. Vuong LT, Mukhopadhyay B and Choi KW: Kinesin-II recruits Armadillo and Dishevelled for Wingless signaling in Drosophila. Development 141: 3222-3232, 2014. 